\title{
Energy Efficient RRM Strategies for Current and Upcoming TeTRA Cellular Systems
}

\author{
Enrico Del Re*, Simone Morosi*, Pierpaolo Piunti*, Giacomo Mazzi*, Ottavio Gremigni ${ }^{\dagger}$ \\ * Department of Information Engineering - University of Florence, Italy \\ e-mail: \{enrico.delre,simone.morosi,pierpaolo.piunti\}@unifi.it, giacomo.mazzi@stud.unifi.it \\ ${ }^{\dagger} \mathrm{LCC}$ consultant at Telecom Italia \\ e-mail: gremigni@gmail.com
}

\begin{abstract}
This paper aims at providing a thorough analysis of the energy efficiency of the TeTRA (TErrestrial Trunked RAdio) private cellular system. In particular some effective dynamic radio resource management solutions are presented, highlighting the impact on daily energy consumption. Moreover, the potential benefits of the transition which can be envisaged to the recently proposed TeTRA over LTE system are evaluated in terms of capacity and energy saving.
\end{abstract}

\section{INTRODUCTION}

In the last decade the ICT companies have started to pay a huge attention to the energy efficiency of the telecommunication stystems due to the compelling need to reduce the energetic bill. As a matter of fact the energetic costs are one of the most affecting drivers in the increasing of operational expenditures (OPEX) [1]. At the same time the reduction of energetic waste means a cut of the greenhouse gas emissions that ends up having a positive effect on the environment.

Therefore, the academic and industrial researchers have recently started to analyze the energy consumption of ICT systems and devices and propose solutions to improve energy efficiency.

Focusing on wireless communications, a lot of results have been presented aiming at improving the energy efficiency of cellular networks by taking into account both the deployment aspects [2] and the dynamic radio resource management (RRM). In particular dynamic RRM is a set of strategies that can help to increase the energy saving in the already deployed cellular networks. In [3] dynamic RRM strategies are classified as:

- frequency domain strategies adapting the available bandwidth to traffic variations by playing with carriers activation and deactivation like in [4] and [5];

- spatial domain strategies adapting the number of antennas at the base station in a MIMO scenario like in [6] or playing with the number of active micro base stations in a heterogeneous scenario like in [7];

- time domain strategies that allow a low power state for the RF stage of the base station during period of no transmission [8].

Moreover, it is worth highlighting that the base station transmission power can be dinamically adapted in order to save energy and guarantee the requested network capacity [9].
Such solutions contribute also to reduce the electromagnetic pollution. Because of their dense diffusion and the envisaged increase of subscribers the analysis of the energy efficiency has been mainly focused on the commercial radio and cellular systems.

As for the institutional contexts, the goal to provide secure, resilient private radio communications has pushed to develop and deploy professional mobile radio (PMR) networks: generally speaking these systems are mainly adopted to provide military and public safety forces a common communication platform and therefore the relative market is mainly reserved to national and regional governments; nonetheless, also some private companies, civil protection and rescue organizations may resort to such systems. Even if the priorities and missions of all these entities are quite different from the ones of the commercial operators and providers, however, in the current scenario of economic crisis and budget cuts the goal of the reduction of the energy costs is a general objective of capital importance: as a result, the evaluation of the energy consumption and efficiency and the definition of energy saving strategies becomes a crucial issue also for the PMR systems.

This paper deals with the energy efficiency of Terrestrial Trunked Radio (TeTRA) cellular system, which is the most common PMR system.

The main contribution of this work is to analyze the power consumption of TeTRA by introducing a power consumption model of TeTRA base station. Moreover, we present some dynamic RRM strategies that could be immediately applied to TeTRA without affecting at all its main features and figures of merits. Finally, the future transition to TeTRA over LTE is evaluated from the energy efficiency point of view.

The paper is organized as follows. In Section II the current TeTRA architecture is presented together with the enhancements in data transmission and the boosters for the future TeTRA over LTE. Then, in Section III the base station power consumption model is described and in Section IV some strategies aiming at improving the energy efficiency are presented for today TeTRA system and for future TeTRA over LTE. Finally Section V concludes the work.

\section{THE TETRA SYSTEM}

TeTRA [10], [11] is a multiple access digital system for secure private radio communications. It allows the transmis- 
sion of high quality voice and data and it has been proposed mainly for emergency services, public safety and in general for all scenarios where a bounded secure area for communications is needed.

TeTRA system introduces some functionalities that cannot be obtained by commercial cellular systems: these features are motivated by the specific purposes of the secure private radio systems with respect to those pursued by wide spread cellular technologies. In particular, TeTRA offers:

- group calls;

- reduced call setup time (below $300 \mathrm{~ms}$ );

- direct mode of operation using other mobile devices as repeater;

- secure data transmission by end-to-end encryption;

- push-to-talk mode.

\section{A. TeTRA architecture}

As depicted in Fig. 1, the TeTRA architecture shows similarities with the generic cellular network ones and presented the following standard interfaces:

- Air Interface (AIR I/F) which ensures the interoperability of terminal equipment of different manufacturers;

- Terminal Equipment Interface (TEI) facilitating the indipendent development of mobile data applications;

- Inter Systems Interface (ISI) which allows the interconnection between TeTRA networks of different manufacturers;

- Direct Mode Operation (DMO) guaranteeing the communication between terminals also beyond network coverage.

A Time Division Multiple Access (TDMA) is used to allocate four channels into one $25 \mathrm{kHz}$ bandwidth carrier. In addition the use of $\pi / 4$-PSK modulation makes the system extremely spectral efficient. Data and control information are mapped into some logical channels which in turn are mapped into physical channels; each physical channel occupies one time slot, i.e. one radio burst, of the downlink radio frame which is described in Fig. 2. At each base station the first slot of each TDMA frame of one carrier is occupied by the Broadcast Common Channel (BCCH). The remaining time slots of such carriers and, eventually, all the time slots of other available carriers can be assigned to a Traffic Channel (TCH). When a channel is idle because it is not assigned to any $\mathrm{TCH}$, a dummy burst is transmitted in order to maintain a continuous bit flow.

\section{B. TeTRA enhancements}

The TeTRA first release was able to provide a $28 \mathrm{kbps}$ datarate which allows the voice service, but guarantees only low quality multimedia transmission. Therefore, a second release of standard has been introduced: the TeTRA Enhanced Data Service (TEDS). TEDS uses different channel bandwidths and data-rates for a flexible use of TeTRA bands. In particular, by supporting four different channel bandwidths from $25 \mathrm{kHz}$ to $150 \mathrm{kHz}$ and different modulation schemes

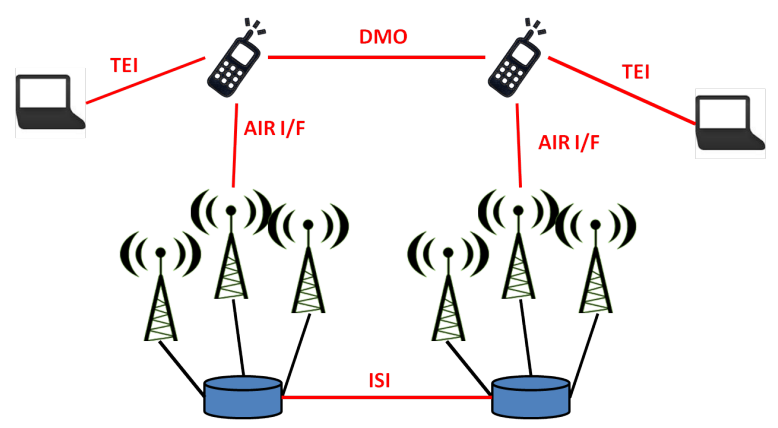

Fig. 1. TeTRA architecture

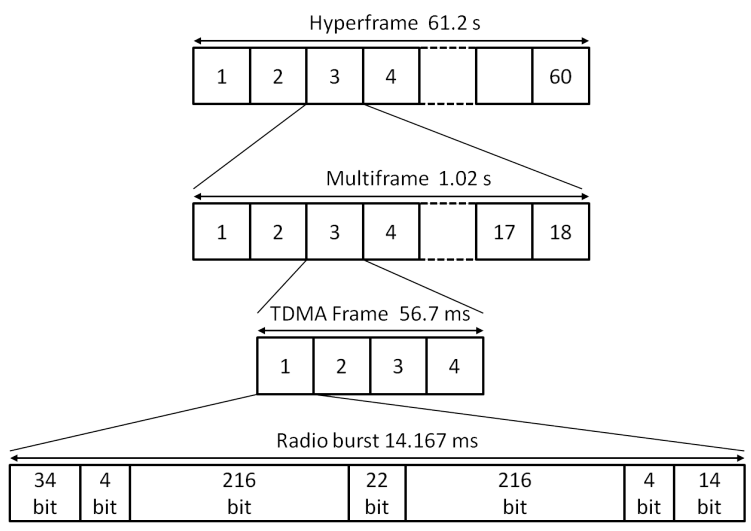

Fig. 2. TeTRA radio frame

( $\pi / 4$-DQPSK, $\pi$ /8-D8PSK, 4-QAM, 16-QAM and 64-QAM), it enables high speed data transmission of about $500 \mathrm{kbps}$.

Moreover, most TeTRA manufacturers are now looking to the future by implementing a TeTRA over LTE system (TeTRAoLTE) [12]. LTE will be the widely deployed global mobile broadband standard; it is characterized by an all-IP system architecture that is supported by a flexible radio interface, guaranteing high data rates and low latency. In addition the LTE system provides some features that meet the PMR requirements, like the possibility to deploy heterogeneous networks, the intercell coordination, the interworking with other radio access technology and the unicast and broadcast service support. On the other hand it is important to stress that some challenges are still to be solved before the implementation of a complete TeTRAoLTE system. In particular resilient and highly available infrastructure, reliable and secure communications, direct communications and group communications are not currently provided by the LTE standard. Nonetheless, since the high data rate allowed by LTE is extremely promising for future TeTRA systems, such challenges are likely to be dealt with by manufacturers and researchers in the next years.

\section{TETRA BASE STATION POWER CONSUMPTION MODEL}

A TeTRA base station is composed of the different blocks which are depicted in the diagram of Fig. 3: the Site Control Unit (SCU) and the Baseband unit (BBU) compose the baseband and management sections while the Radio Frequency Unit (RFU) and the Antenna Switching Circuit (ASC) are 


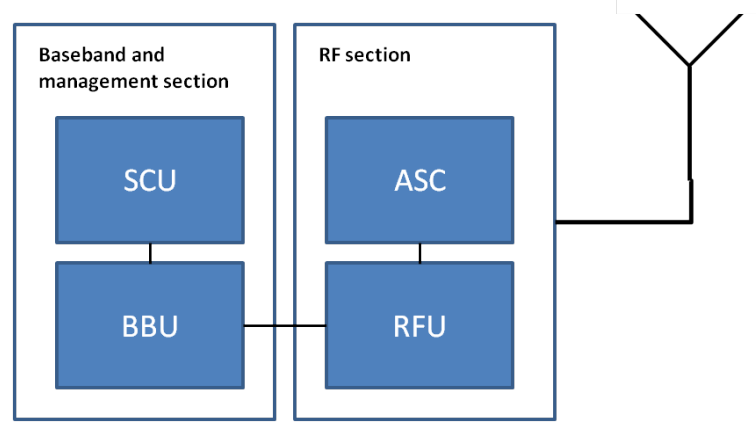

Fig. 3. TeTRA BS Structure

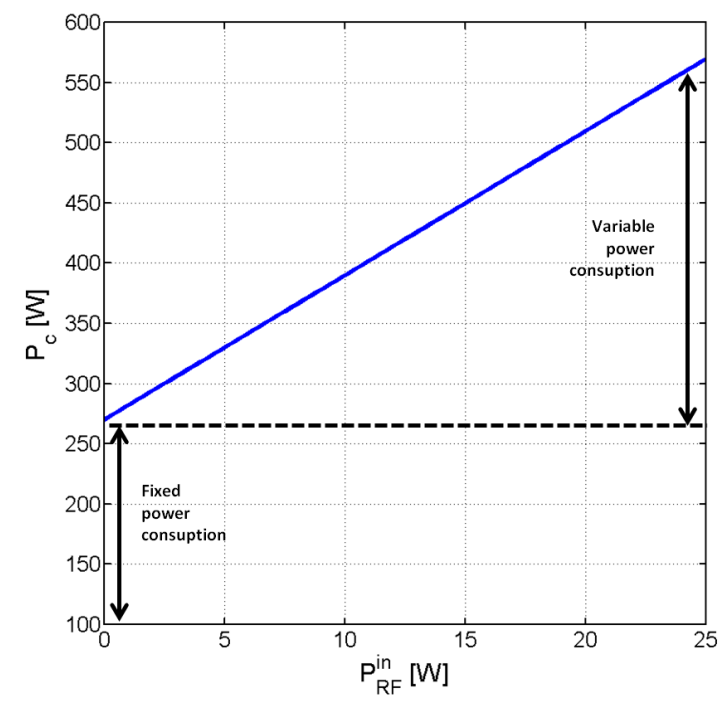

Fig. 4. TeTRA power consumption model

dedicated to the radio frequency section: the SCU and BBU are responsible for the management operations of the considered site and for the baseband signal processing operations. The number of SCU and BBU is variable and depends on the desired redundancy level. The RFU controls the RF operations on the transmitted and received signal and the ASC encompasses the circuits which connect the RFU modules to the antenna system. The number of RFU depends on the number of carriers that are available in the site.

A power consumption model for the TeTRA base station can be obtained by properly adapting the models of the other commercial cellular systems. In particular, we use as a starting point the linear model which is considered in [13]. The linear model is composed of a fixed and a variable component. The fixed part models the power consumption that is absorbed independently from the traffic managed by the BTS. The variable part models the RF power consumption and assumes different values depending on the RF power at the antenna port. Such model can be written as follows:

$$
P_{c}=n_{R F U} \cdot P_{R F U}+P_{B B U}+P_{S C U}+P_{A S C}
$$

where $n_{R F U}$ is the number of Radio Frequency Units (RFU), each one consuming $P_{R F U} \mathrm{~W} ; P_{B B U}, P_{S C U}$ and $P_{A S C}$ are respectively the power consumption of BBU, SCU and ASC. In particular, also $P_{R F U}$ contains a fixed and a variable part:

$$
P_{R F U}=\alpha \cdot P_{R F}^{i n}+P_{R F}^{0}
$$

where $P_{R F}^{i n}$ is the RF power at the antenna port and $P_{R F}^{0}$ is the fixed power consumption that RFU absorbs when there is no power at the antenna port. Hence, a final expression of the power consumption model can be derived as

$$
P_{c}=\alpha \cdot P_{R F}^{i n}+P_{0}
$$

Taking into account some manufacturers datasheets, we consider $\alpha=12$ and $P_{0}=274 \mathrm{~W}$.

\section{ENERGY EFFICIENCY IN TETRA}

In this section the main strategies that could be applied in order to obtain a more efficient TeTRA system are introduced. In particular we focus on the resource management strategies without forgetting that to perform a simple replacement of the older BS devices with new hardware blocks will introduce a significant energy saving. Like in a GSM system, the resource management strategies can be discriminated by identifying whether they refer or not to the $\mathrm{BCCH}$ carrier. While the $\mathrm{BCCH}$ carrier should be always on air in order to transmit the broadcast control information, the other carriers could be put on a energy saving state when no TCHs are allocated. Since such feature is already implemented in some TETRA systems we focus on the efficiency of $\mathrm{BCCH}$ carrier consumption depending on the effects of the power control. Finally, we introduce a visionary approach that considers the potential increase of energy efficiency due to the introduction of the TeTRAoLTE system.

\section{A. Power control on BCCH transceiver}

Power Control is a software based solution that allows an energy saving mode on the $\mathrm{BCCH}$ transceiver. Such a solution reduces the overall power consumption by transmitting dummy bursts on the idle channels, i.e. on the time slots that are not allocated to a $\mathrm{TCH}$, with a power level lower than the maximum power of the $\mathrm{BCCH}$ channel. The behaviour of Power Control is shown in Fig. 5: note that in our analysis a 2 $\mathrm{dB}$ power reduction has been considered. Instead, the $\mathrm{BCCH}$ channel is transmitted at full power in order to keep the cell range unaltered. Therefore, the power Control allows to make the power consumtpion variable according to the served traffic. Fig. 6 shows the behaviour of TETRA BS power consumption when Power Control is and is not applied. The highest saving is achievable when the traffic is very low; for higher traffic values the Power Controlled results converge to the baseline solution ones.

\section{B. A step ahead: TeTRAoLTE}

As explained in Section II, the transition to a TETRA over LTE system is actually under investigation. LTE would guarantee high quality communications due to the OFDM technology and adaptive modulation and coding and a better energy management due to the hardware efficiency and to the 


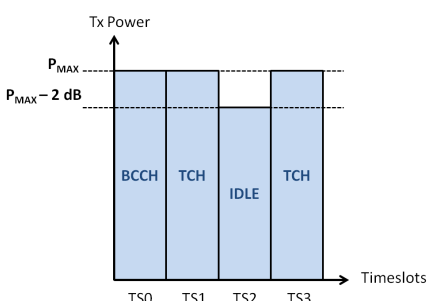

(a) Power control

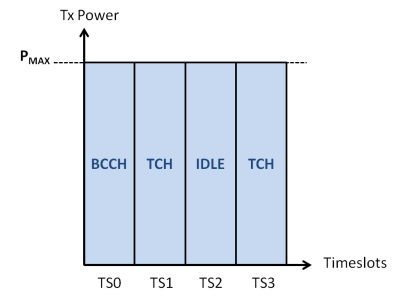

(b) No power control
Fig. 5. Transmitted signals for power control (a) and no power control (b) cases

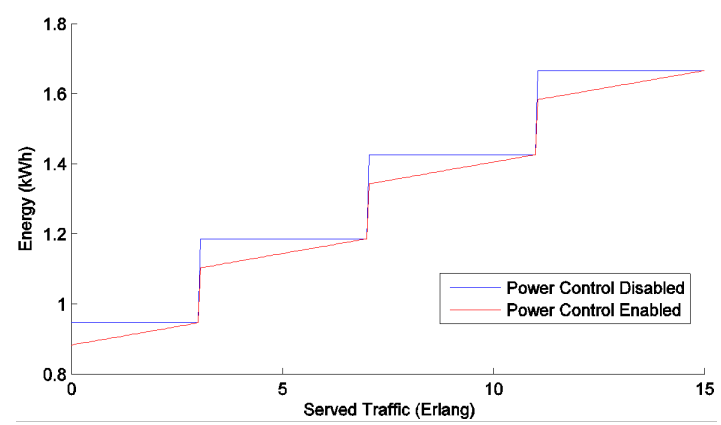

Fig. 6. BS power consumption versus served traffic for the power control and no power control cases

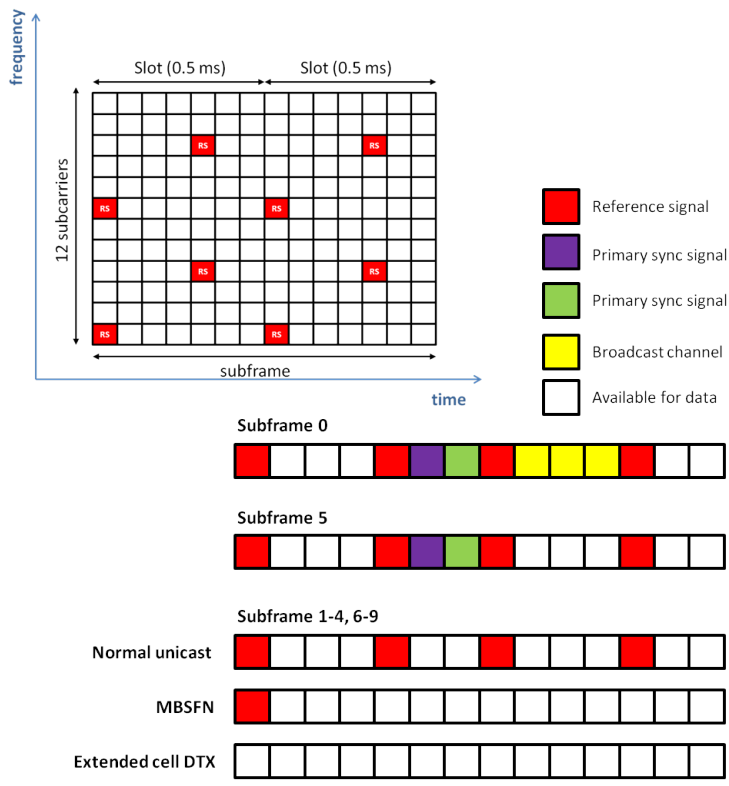

Fig. 7. Cell DTX: PAs can be put in low power state during the available slots if there is no downlink traffic

possibility to implement dynamic RRM techniques. As for the energy saving, the most promising among the enabling techniques in a LTE base station is the cell DTX (Discontinous Transmission). Cell DTX is a time domain energy saving strategy which allows a low power state (sleep mode) for the RF section during the time with no downlink transmission. Thereby, the cell coverage is not affected by the sleep mode since the reference symbols are always transmitted at the same

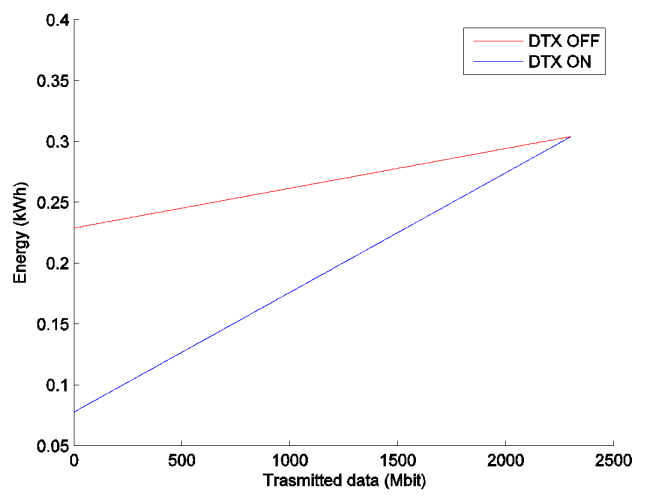

Fig. 8. BS power consumption versus hourly served traffic in the case of DTX and no DTX for TETRAoLTE

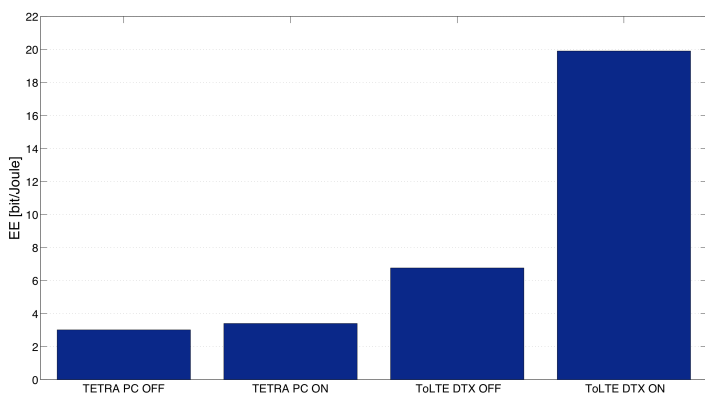

Fig. 9. Energy efficiency for the considered TeTRA systems

power level. As shown in Fig. 7 only a small fraction of each subframe must be transmitted even if the base station is not managing any traffic flow. In particular a high cell DTX gain can be obtained using MBSFN or extended cell DTX subframes instead of the normal unicast ones [14].

The energy consumption of a LTE base station with cell DTX is [15]:

$$
E_{h}=\left(\alpha P_{t x}+P_{0}\right) \eta_{h}+\epsilon P_{\text {sleep }}\left(1-\eta_{h}\right)+(1-\epsilon) P_{0}\left(1-\eta_{h}\right)
$$

where $\alpha$ is the slope of the dynamic power consumption, $P_{t x}$ is the power that is transmitted by the $\mathrm{BS}, P_{0}$ is the fixed power consumption when the $\mathrm{BS}$ is active, $P_{\text {sleep }}$ is the power consumption during the DTX time and $\epsilon$ is a binary variable modelling whether DTX is active or not. Typical value for an LTE system are: $\alpha=4.7, P_{0}=210 \mathrm{~W}$ and $P_{\text {sleep }}=21 \mathrm{~W}$. The hourly load $\eta_{h}$ can be expressed as

$$
\eta_{h}=\frac{\frac{D_{h}}{T}}{3600}+\eta_{s}
$$

where $D_{h}$ is the total amount of data to be transmitted at hour $h, T$ is the maximum allowed system datarate and $\eta_{s}$ is the load of signaling traffic, i.e., of the pilot symbols.

In this analysis we assume the bandwidth equal to $1.4 \mathrm{MHz}$ and a fixed $20 \mathrm{~W}$ transmission power. The maximum allowed datarate is $0.8 \mathrm{Mbps}$. Fig. 8 shows the impact of the cell DTX on the power consumption of an LTE base station. When the cell DTX is not enabled the power consumption shows limited variations depending on the transmitted data values: this trend 
can be explained by recalling that the BTS has a fixed power consumption even if the transmitted power is zero, i.e., no transmission is performed. On the other hand, when cell DTX is enabled the power consumption is reduced of about $50 \%$ when low data loads are transmitted and converges to the no DTX case for full frame transmission.

Finally, in Fig. 9 the comparison of the energy efficiency of the different considered TeTRA systems is shown. By making use of the well know bit/Joule metric, the energy efficiency is calculated as follows:

$$
E E=\frac{\sum_{h} D_{h}}{\sum_{h} E_{h}} \quad[\text { bit/Joule }]
$$

where $D_{h}$ is the amount of downlink data at certain hour $h$ of the day and $E_{h}$ is the relative energy consumption. Figure 9 shows the increase of the energy efficiency which is due to the proposed solutions with respect to the actual system. More in detail, effective hardware solutions and the cell DTX flexible implementation drastically reduce the energy impact the of TeTRA cellular networks. Note that these efficient solutions also allow maximum site capacity. It is worth highlighting that the $\mathrm{BCCH}$ power control slightly improve network energy efficiency.

\section{CONCLUSION}

In this paper the energy efficiency of the TeTRA cellular system has been considered. First of all a base station power consumption model has been proposed by considering the modules constituting a generic TeTRA BTS. Then, some energy saving solutions have been evaluated. In particular, the $\mathrm{BCCH}$ power control allows the reduction of transmitted power during the timeslots when the BTS is not transmitting traffic data. The strength of such solutions is to improve the TeTRA energy efficiency without any significant modification in the architecture. Finally, a possible transition to the TeTRA over LTE system has been considered, showing that the TeTRAoLTE solution would increase both the capacity and the energy efficiency because of the effectiveness of both the hardware devices and the radio resource management flexibility.

\section{REFERENCES}

[1] C. Bianco, F. Cucchietti, and G. Griffa, "Energy consumption trends in the next generation access network, a telco perspective," in Telecommunications Energy Conference, 2007. INTELEC 2007. 29th International, Sept 2007, pp. 737-742.

[2] S. Morosi, A. Fanfani, and E. Del Re, "Network deployment and rrm strategies for green mobile communications," in European Wireless, 2012. EW. 18th European Wireless Conference, 2012, pp. 1-7.

[3] T. Chen, H. Zhang, Z. Zhao, and X. Chen, "Towards green wireless access networks," in Communications and Networking in China (CHINACOM), 2010 5th International ICST Conference on, Aug 2010, pp. $1-6$.

[4] L. Saker, S.-E. Elayoubi, and T. Chahed, "Minimizing energy consumption via sleep mode in green base station," in Wireless Communications and Networking Conference (WCNC), 2010 IEEE, April 2010, pp. 1-6.

[5] S. Morosi, P. Piunti, and E. D. Re, "Sleep mode management in cellular networks: a traffic based technique enabling energy saving," Transactions on Emerging Telecommunications Technologies, vol. 24, no. 3, pp. 331-341, 2013. [Online]. Available: http://dx.doi.org/10.1002/ett.2621
[6] P. Skillermark and P. Frenger, "Enhancing energy efficiency in lte with antenna muting," in Vehicular Technology Conference (VTC Spring), 2012 IEEE 75th, May 2012, pp. 1-5.

[7] S. Morosi, P. Piunti, and E. Del Re, "Improving cellular network energy efficiency by joint management of sleep mode and transmission power," in Digital Communications - Green ICT (TIWDC), 2013 24th Tyrrhenian International Workshop on, Sept 2013, pp. 1-6.

[8] P. Frenger, P. Moberg, J. Malmodin, Y. Jading, and I. Godor, "Reducing energy consumption in lte with cell dtx," in Vehicular Technology Conference (VTC Spring), 2011 IEEE 73rd, May 2011, pp. 1-5.

[9] S. Morosi, P. Piunti, and E. Del Re, "A forecasting driven technique enabling power saving in lte cellular networks," in IEEE 9th International Conference on Wireless and Mobile Computing, Networking and Communications (WiMob), 1st Workshop on GReen Optimized Wireless Networks (GROWN'13), 2013, Oct 2013, pp. 217-222.

[10] "Terrestrial Trunked Radio (TETRA); Voice plus Data (V+D); Designers' guide; Part 1: Overview, technical description and radio aspects," ETSI Technical Report 300-1, 1997.

[11] "Terrestrial Trunked Radio (TETRA); Voice plus Data (V+D); Part 2: Air interface," ETSI Technical Report 392-2, 2001.

[12] T. Doumi, M. Dolan, S. Tatesh, A. Casati, G. Tsirtsis, K. Anchan, and D. Flore, "Lte for public safety networks," Communications Magazine, IEEE, vol. 51, no. 2, pp. 106-112, February 2013.

[13] "Energy efficiency analysis of the reference systems, areas of improvements and target breakdown," EARTH project deliverable D2.3, 2010.

[14] T. Chen, Y. Yang, H. Zhang, H. Kim, and K. Horneman, "Network energy saving technologies for green wireless access networks," Wireless Communications, IEEE, vol. 18, no. 5, pp. 30-38, October 2011.

[15] S. Tombaz, S. Han, K. Sung, and J. Zander, "Energy efficient network deployment with cell DTX," pp. 1-1, 2014. 